\title{
A Comparative Study and Analysis of an Optimized Control Strategy for the Toyota Hybrid System
}

\author{
Theo Hofman ${ }^{1}$, Thijs Purnot \\ ${ }^{I}$ Author 1 (corresponding author) Technische Universiteit Eindhoven, \\ P.O. Box 513, 5600 MB EINDHOVEN, The Netherlands, \\ Phone: +31402472798,Fax:+31402461418,E-mail: t.hofman@tue.n
}

\begin{abstract}
The Toyota Prius equipped with the Toyota Hybrid System (THS) II vehicle uses a combination of a combustion engine and two electric machines in order to increase the efficiency and the fuel economy. The Energy Management Strategy (EMS) of the THS II is analyzed using measurement data collected with a Toyota Prius under different driving conditions on the road and compared with an optimized EMS based on simulations. The main goal of this paper is to verify the control strategy as implemented in the Prius by comparing the measured strategy with an optimized strategy based on Energy Consumption Minimization Strategy (ECMS). The differences between the simulation results and the actual measured strategy are analysed and discussed.
\end{abstract}

Keywords: Hybrid Electric Vehicles, Modelling and Simulation, Hybrid Strategy, Energy Efficiency, Energy Consumption, Power Train, Transmission, Optimal Control

\section{Introduction}

The Toyota Prius (see, Figure 1) equipped with the Toyota Hybrid System (THS) II vehicle uses a combination of a combustion engine and two electric machines in order to increase the efficiency and the fuel economy $[1,2,3,4,6,7]$. The Energy Management Strategy (EMS) of the THS II is analyzed using measurement data collected with a Toyota Prius under different driving conditions on the road and compared with an optimized EMS based on simulations. The main goal of this paper is to verify the control strategy as implemented in the Prius by comparing the measured strategy with an optimized strategy based on Energy Consumption Minimization Strategy (ECMS) [3].

The outline of the paper is as follows. In Section 2 , the vehicle model is discussed including the control model. In Section 3, the simulation approach is discussed.

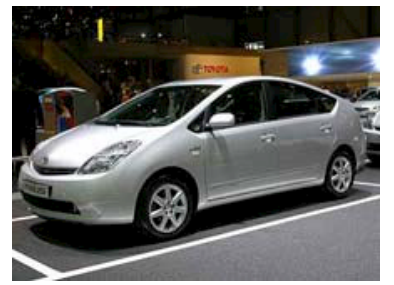

Figure 1: Toyota Prius equipped with the THS II

Accordingly, in Section 4 the simulation results by comparing the fuel economy results with the catalogues values on two drive cycles are described. Additionally, in Section 4, the control strategy of the model is further optimized using actual measurement data of the Toyota Prius under different driving circumstances. This is performed using a defined optimization criterion, which minimizes the optimal engine operation points 
computed with the simulation model and known control strategy and the measured operation points with unknown control optimization algorithm implemented in the Toyota Prius. Finally, the conclusions are described in Section 5.

\section{Vehicle Simulation Model}

The vehicle simulation model is a backwards control model [3]. The requested vehicle drive power $P_{d}$ and requested vehicle speeds $\omega_{d}$ are the inputs of the model. These inputs are used to calculate the optimal engine output torque $T_{e}^{o}$ and speed $\omega_{e}^{o}$ set points. Figure 2 shows a schematic overview of this model. The battery power $P_{b a t}$ is determined using the generator and electric motor power.

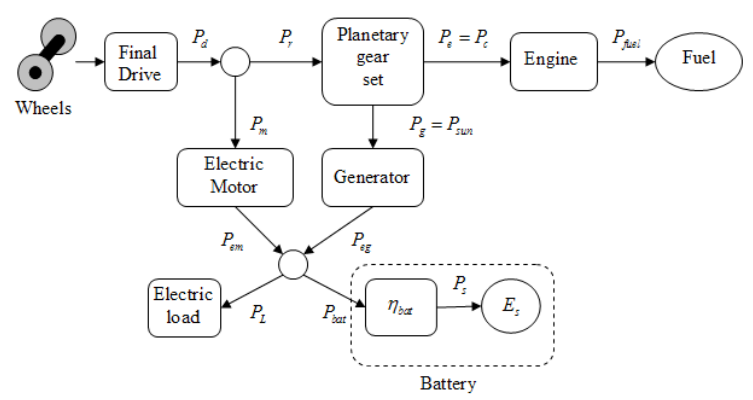

Figure 2: Power-based control model for the THS [3]

\subsection{Dynamic drive train model}

In Table 1 an overview is given of the sequence of calculation for the engine torque $T_{e}$ and speed $\omega_{e}$ based on a given motor $T_{e m}$ and generator $T_{e g}$, vehicle drive torque $T_{d}$, and angular vehicle speed $\omega_{d}$. The optimal value for the electric machine torques follow from the optimal control strategy, which is discussed in Section 2.2.

The relation between the acceleration of the ring gear $\dot{\omega}_{r}$ (which is directly related to the angular vehicle acceleration $\dot{\omega}_{d}$ ) and the torques in the transmission:

$$
\begin{aligned}
& \left(\frac{I_{m}^{\prime}\left(N_{r}+N_{s}\right)^{2}}{N_{r} I_{e}^{\prime} z_{f d}}+\frac{N_{s}^{2} I_{m}^{\prime}}{N_{r} I_{g}^{\prime} z_{f d}}+N_{r}\right) \dot{\omega}_{r}= \\
& \left(\frac{\left(N_{r}+N_{s}\right)^{2}}{N_{r} I_{e}^{\prime}}+\frac{N_{s}^{2}}{N_{r} I_{g}^{\prime}}\right) T_{m}+\frac{\left(N_{r}+N_{s}\right)}{I_{e}^{\prime}} T_{e},(1) \\
& +\frac{N_{s}}{I_{g}^{\prime}} T_{g}-\left(\frac{\left(N_{r}+N_{s}\right)^{2}}{N_{r} I_{e}^{\prime} z_{f d}}+\frac{N_{s}^{2}}{N_{r} I_{g}^{\prime} z_{f d}}\right) \frac{F_{d} R_{\text {tire }}}{\eta_{f d}}
\end{aligned}
$$

where $I_{v}^{\prime}$ is the lumped inertia of the ring gear $I_{r}$ and the electric motor $I_{m}, I_{g}^{\prime}$ the lumped inertia of the sun gear $I_{s}$ and the generator $I_{g}$ and $I_{e}^{\prime}$ the lumped inertia of the combustion engine $I_{e}$ and the carrier gear $I_{c}$ :

$I_{v}^{\prime}=I_{m}+I_{r}$,

$I_{g}^{\prime}=I_{g}+I_{s}$,

$I_{e}^{\prime}=I_{e}+I_{c}$.

Table 1: Sequence of calculation for the engine torque and speed based on a given motor and generator, vehicle drive torque, and vehicle speed

1. Given the described vehicle speed, the ring gear acceleration is determined and the ring speed change holds $\dot{\omega}_{r}=\frac{d}{d t} \omega_{r}$.

2. For the chosen values of $T_{e g}$ and $T_{e m}$ and the prescribed value of $T_{d}$, the engine torque $T_{e}$ is determined using (1).

3. Given the chosen values of $T_{e g}$ and $T_{e m}$ and the prescribed value of $T_{d}$, the engine acceleration $\dot{\omega}_{e}$ and engine speed $\omega_{e}$ are determined using (6).

4. Using the ring gear speed $\omega_{r}$ and the engine speed $\omega_{e}$, the generator speed is calculated using $\omega_{g}+\omega_{r} z_{p g s}=\omega_{e}\left(z_{p g s}+1\right)$ with planetary gear ratio denoted as $z_{p g s}$.

5. Finally, the generator and electric motor power are determined using the calculated torques and speeds of the electric machines.

The planetary gear ratio $z_{p g s}$ is defined by the radii of the ring gear and sun gear or by the number of teeth of the ring gear $\left(N_{r}\right)$ and the sun gear $\left(N_{s}\right)$ : 
$z_{p g s}=\frac{R_{r}}{R_{s}}=\frac{N_{r}}{N_{s}}$.

Moreover, the final drive efficiency, wheel tyre radius, the drive force are denoted as $\eta_{f d}, R_{\text {tire }}$ and $F_{d}$ respectively.

The relation between the engine acceleration and the torques in the drive train is given by:

$\left(\frac{N_{r}^{2} z_{f d} I_{e}^{\prime}}{\left(N_{r}+N_{s}\right) I_{m}^{\prime}}+\frac{N_{s}^{2} I_{e}^{\prime}}{\left(N_{r}+N_{s}\right) I_{g}^{\prime}}+\left(N_{r}+N_{s}\right)\right) \dot{\omega}_{e}=$ $\left(\frac{N_{r}^{2} z_{f d}}{\left(N_{r}+N_{s}\right) I_{m}^{\prime}}+\frac{N_{s}^{2}}{\left(N_{r}+N_{s}\right) I_{g}^{\prime}}\right) T_{e}$ $+\frac{z_{f d} N_{r}}{I_{m}^{\prime}} T_{m}-\frac{N_{s}}{I_{g}^{\prime}} T_{g}-\frac{N_{r}}{I_{m}^{\prime}} \frac{F_{d} R_{\text {tire }}}{\eta_{f d}}$

In this paper, the engine and ring gear speed, which are related to the vehicle speed via the planetary gear, are used as the two states.

\subsection{Control Model}

The Energy Management Strategy EMS used for the dynamic control model is based on the Energy Consumption Minimization Strategy (ECMS) [3]. The ECMS is used to find an optimal set point for the electric machines powers in each transmission operating point. Knowing the torques and speeds in the transmission, the ECMS [3] is applied to find the optimal set point of the generator power $P_{e g}$ and electric motor power $P_{e m}$.

$$
\left(P_{e m}^{o}, P_{e g}^{o}\right)=\left\{\begin{array}{l}
\left(0, P_{e m}^{R}\right) \text { if } P_{d}<0 \\
\left(0, P_{e m}^{M O}\right) \text { if } f^{M O}<f^{P S} \wedge \\
0 \leq P_{d} \leq P_{e m \max } \wedge P_{e m}^{M O}<P_{b a t \max } \\
\left(P_{e m}^{P S}, P_{e g}^{P S}\right) \text { elsewhere }
\end{array}\right.
$$

with the electric motor power during the MotorOnly (MO) driving mode:

$P_{e m}^{M O}=\frac{P_{d}}{\eta_{e m}}+P_{L}$,

and the Regenerative (R) braking mode:

$P_{e m}^{R}=s a t\left[f_{b f d} \times P_{d}\right]_{0}^{P_{e m \max }}$,

where $f_{b d f}[-]$ represents the brake power distribution factor.
$\left(P_{e m}^{P S}, P_{e g}^{P S}\right)=\arg \min _{\substack{\left(P_{e m}, P_{e g}\right) \\ \in Q}}\left\{f^{P S}\left(P_{e m}, P_{e g} \mid T_{d}, \omega_{d}\right)\right\}$

where $Q$ covers the set of constraints for the drive train components:

$$
\begin{aligned}
& Q= \\
& \left\{\begin{array}{l}
\left(T_{e g}, T_{e m}\right) \mid \omega_{e \text { min }}(t) \leq \omega_{e}(t) \leq \omega_{e \text { max }}(t) \\
\omega_{e g \text { min }}(t) \leq \omega_{e g}(t) \leq \omega_{e g \text { max }}(t) \\
\omega_{e m \text { min }}(t) \leq \omega_{e m}(t) \leq \omega_{e m \text { max }}(t) \\
T_{e, \text { min }}(t) \leq T_{e}(t) \leq T_{e, \text { max }}(t) \\
T_{e g \text { min }}(t) \leq T_{e g}(t) \leq T_{e g \text { max }}(t) \\
T_{e m \text { min }}(t) \leq T_{e m}(t) \leq T_{e m \text { max }}(t) \\
P_{e g \text { min }}(t) \leq P_{e g}(t) \leq P_{e g \text { max }}(t) \\
P_{e m \text { min }}(t) \leq P_{e m}(t) \leq P_{e m \text { max }}(t) \\
P_{b a t \text { min }}(t) \leq P_{e g}(t)+P_{e m}(t)+P_{L} \leq P_{b a t \text { max }}(t)
\end{array}\right\}
\end{aligned}
$$

The equivalent fuel cost for driving electrically is:

$f^{M O}=\frac{1}{\eta_{b a t}} P_{e m}^{M O} \cdot \lambda$,

and the equivalent fuel costs for charging or motor-assisting during driving is given by

$f^{P S}=f\left(P_{e m}, P_{e g} \mid T_{d}, \omega_{d}\right)-\lambda \cdot P_{s}^{P S}$,

where $\lambda$ represents the equivalent fuel cost factor in $[\mathrm{g} / \mathrm{J}]$. For the power-split mode (PS) a dense grid of electric machine powers is used to find the optimal set point $\left(P_{e m}^{P S}, P_{e g}^{P S}\right)$. The advantage of using a grid in the optimization procedure is that no requirements are posed for convexity of the objective function. However, in case there does not exist a unique global minimum, the solution with the lowest battery activity is selected. The optimization routine is repeated using variable values of the equivalent fuel $\cos$ factor $\lambda$, until the optimal value $\lambda^{o}$ is found. For $\lambda^{o}$, the system is charge sustaining over the chosen drive cycle..

\section{Simulation Approach}

Using the dynamic equations for the drive train and the control model from Section 2 and the specifications of the THS II [1,2,6,7], a simulation model is build in a Matlab/Simulink environment. The vehicle block in the model (see, Figure 3) contains the vehicle parameters. The block is used to calculate the requested vehicle drive torque and speed using the prescribed vehicle speed that follows from the drive cycle block. 


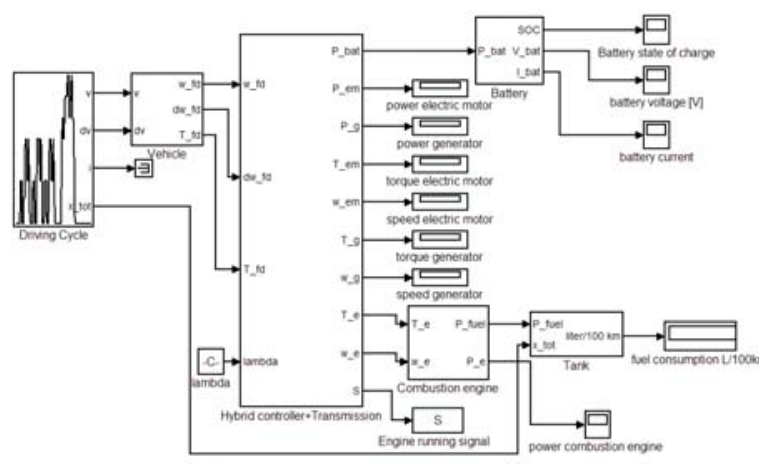

Figure 3: Simulation model for the THS

The hybrid controller block contains the ECMS optimization strategy, as discussed in Section 2. Therefore, the dynamic equations and the parameters for the generator, electric motor, final drive and planetary gear set are included in the hybrid controller block. Furthermore, the hybrid controller is divided into three parts that describe the different drive modes, which can occur: (i) power-split, (ii) electric-drive and (iii) brake energy recovery mode. The combustion engine block and tank block are used for determining the fuel consumption corresponding to the engine speed and torque that follow from the hybrid controller. The battery state of charge, voltage and current are also computed.

\section{Simulation Results}

The fuel consumption over two reference drive cycle (JP10-15, NEDC) has been computed and compared with the catalogue values (see, Table 2). The transmission speed ratio with the baseline model is controlled maximizing the system efficiency whereby the battery power flow is zero over the whole cycle. It can be seen that there is a difference of $9.1 \%$ and $11.9 \%$ between the catalogue and the simulated values on the JP1015 and the NEDC cycle respectively. A drive train parameter variation $(+/-5 \%)$ study has been performed in order to investigate if the model assumptions (final drive efficiency, vehicle mass, brake force distribution) were sufficiently correct. It was found that large variation of drive train parameters were required to reduce the discrepancy and the fuel economy sensitive to parameter variation was relative low. Therefore, it is more likely that differences in fuel economy between the ECMS model and the THS II vehicle are caused by differences in energy management strategy. This assumption will be investigated next.
Table 2: Simulation results

\begin{tabular}{lcccc}
\hline Model & \multicolumn{4}{c}{ Fuel Consumption [1/100km] } \\
\cline { 2 - 5 } & JP10-15 & $\begin{array}{c}\text { Compared } \\
\text { to baseline } \\
{[\%]}\end{array}$ & NEDC & $\begin{array}{c}\text { Compared } \\
\text { to baseline } \\
{[\%]}\end{array}$ \\
\hline Baseline & 5.5 & 100.0 & 5.2 & 100.0 \\
ECMS & 3.6 & 65.7 & 4.0 & 77.0 \\
THS II & 3.1 & 56.6 & 3.4 & 65.1 \\
\hline
\end{tabular}

\subsection{Comparison between measured control strategy and ECMS for the THS II using a constant $\lambda$}

In order to make a comparison between the measured control strategy and the optimized, vehicle data from measurement tests performed on a THS II vehicle are used. The data was obtained using a CAN-bus reader that was connected to the CAN-bus connector of the THS II vehicle. Using the CAN-bus reader, vehicle data can be directly sent to a computer where it is processed. The CAN-bus data is obtained from the internal vehicle sensors. The signals from these sensors are also used by the vehicle energy management system. In total nine signals are read using the CAN-bus reader. These nine signals are measured for ten test drive cycles, each containing different driving situations. The following figure shows the relevant vehicle data that is used to analyze the energy management strategy of the THS II vehicle. The measured signals are indicated in black. The signals that are calculated using the measured signals are illustrated in gray.

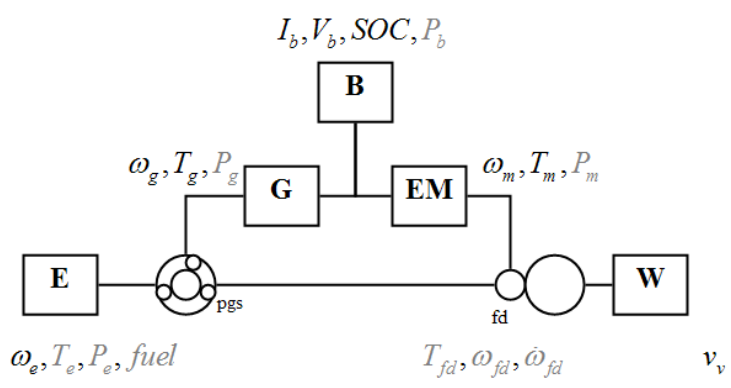

Figure 4: Simulation model for the THS

Figure 4 shows that also the generator and electric motor torque are read from the CAN-bus. The torque signals from the CAN-bus are determined using the current and/or voltage signals from the electric machines. The signals are measured for ten different driving situations: six short time period and four long time period measurements, which can each be considered as drive cycles. The three most suitable measured driving situations that will 
be analyzed are illustrated in Figure 5, where the vehicle speed for each situation is plotted.
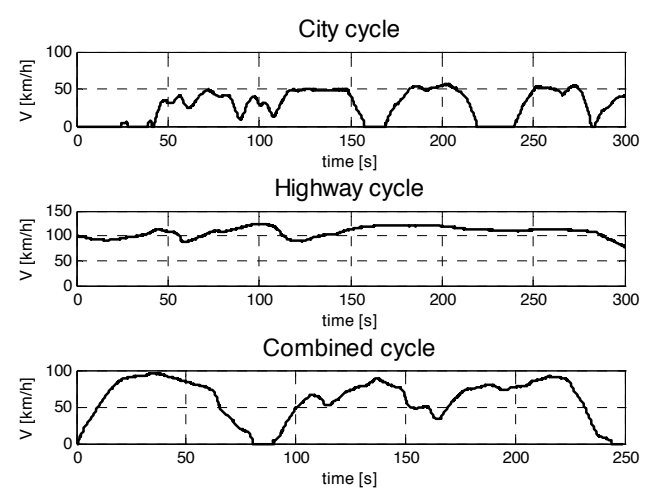

Figure 5: Measured speeds for the three measured drive cycles that will be analyzed in this paper.

The constant equivalent fuel $\cos t \lambda$ (in the next section a variable will be investigated) is chosen such that the end value of the battery energy for the simulation model equals the end value of the measurement data. The influence of the equivalent fuel cost factor $\lambda$ on the difference in battery energy end values between the simulation model and the measured data is illustrated in Figure 6 for the three different drive cycles shown in Figure 3 . The values of $\lambda$ that result in a difference in battery energy end values between the simulation model and the measured data equal to zero (City: $\lambda=3.27$, Highway: $\lambda=3.07$, Combined: $\lambda=3.25$ ) were used in the simulation model for generating the results as shown in Table 3 .

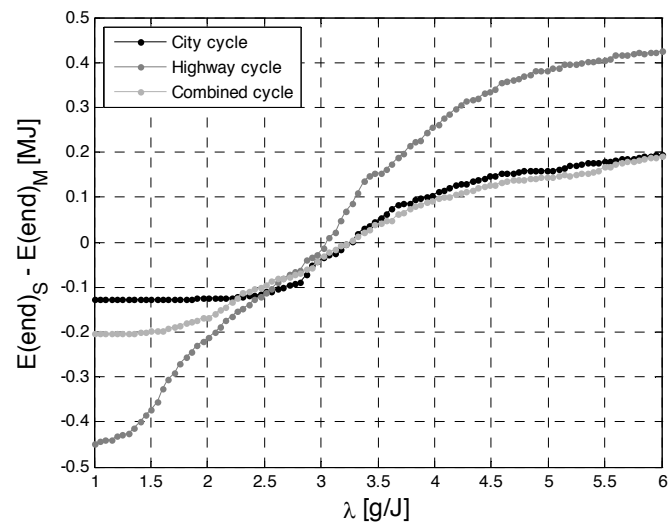

Figure 6: Difference in end values of battery energy between the measured data $(\mathrm{M})$ and the simulation model (S) as a function of the equivalent fuel cost factor $\lambda$.
In the same table the fuel consumption results are shown using the measure engine speed and computed engine speed based on measurement data. For computing the fuel consumption based on the measured vehicle states as shown in Figure 2, the same component efficiency data is used as for the simulation model. In Figure 7, 8 and 9 some simulated and measured results on the city drive cycle are shown.

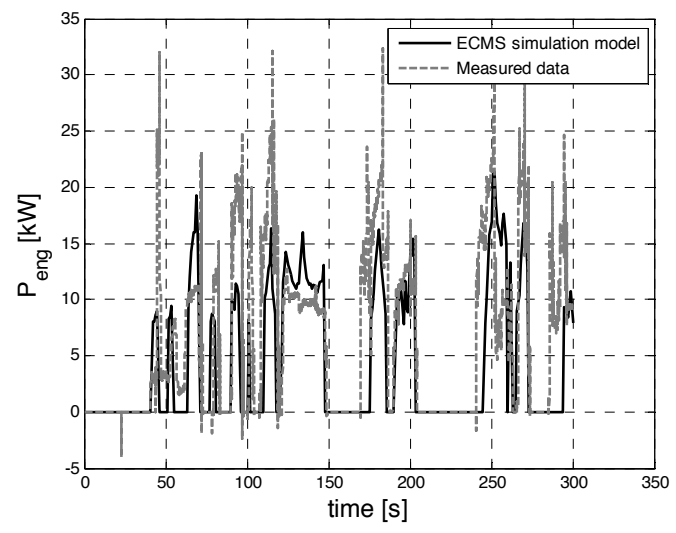

Figure 7: Engine power $P_{e}$ as function of time for the simulation model and measured vehicle data (city drive cycle (see, Figure 3, top diagram)).

Significant differences in engine power between the measured vehicle data and the simulation model data are noticeable for all three measured drive cycles. The state-of-energy SOE signals for the simulation model and the measured vehicle data are illustrated in Figure 8.

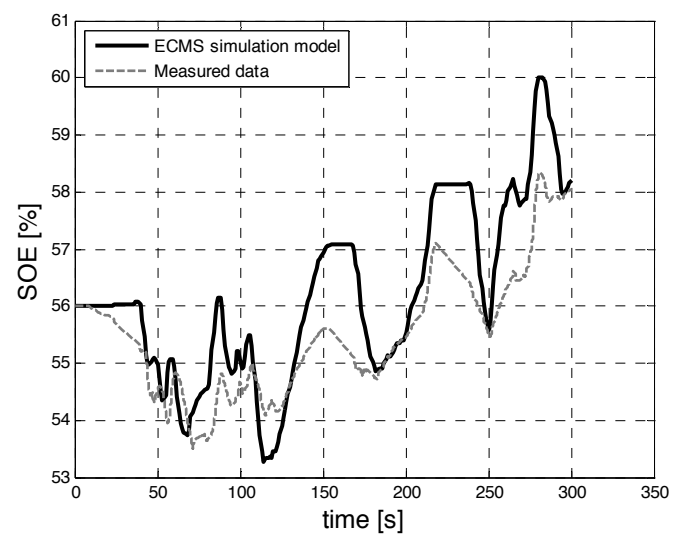

Figure 8: Battery state-of-energy as a function of time for the simulation model and the measured data (city drive cycle).

Figure 8 shows that the measured THS II state-ofenergy less fluctuate than the state-of-energy computed with the simulation model. Table 3 shows that the fuel consumption for the simulation model shows a small benefit for the highway cycle $(2.4 \%)$ and the combined cycle $(6.8 \%)$ compared 
to the measured fuel consumption values. For the city cycle, the benefits in fuel consumption are much higher (36\%).

Table 3: Fuel consumption using simulation model, the measured vehicle data

\begin{tabular}{lccc}
\hline & \multicolumn{3}{c}{ Fuel consumption } \\
& City & Highway & Comb. \\
\cline { 2 - 4 } & 4.83 & 5.57 & 5.42 \\
Simulation & 7.26 & 5.59 & 5.67 \\
Measurement & & & \\
\hline
\end{tabular}

The low fuel consumption is partially caused by the defined ECMS, which assumes that the vehicle can switch from electric drive mode to power split mode instantly. The engine operates at the desired speed and torque level immediately and therefore always in the most efficient area. In reality, the combustion engine has to be cranked and reveled up before switching to power-split mode can occur. The engine can not operate in its most efficient area immediately. In Figure 9, the engine operating points are plotted for the simulation model and the measured data.

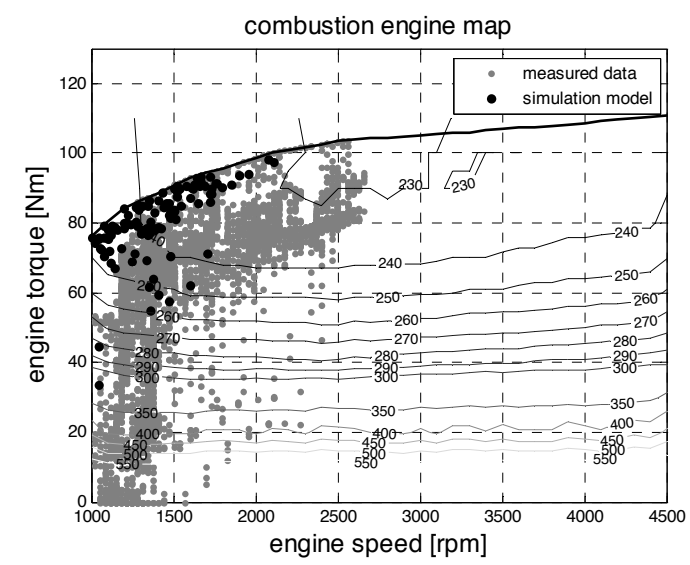

Figure 9: Engine operating points for simulation model and measured vehicle data for the city drive cycle.

Although, the difference in fuel consumption between the simulation model and the measured vehicle are relative small for the highway and combined cycle, the difference for the city cycle is large. Furthermore, difference in engine operation and battery power flow is found to be large. The ECMS in this section was based on a constant equivalent fuel cost factor in the next section a variable equivalent fuel cost factor is optimized using a cost criterion minimizing the computed engine operation points with the simulation model and the measured engine operation points.

\subsection{Comparison between measured control strategy and ECMS for the THS II using a variable $\lambda$}

In order to improve the accuracy of the ECMS simulation model and decrease the differences compared to the measured vehicle data, the influence of a time depend variable equivalent fuel cost factor $\lambda(t)$ is investigated. The variable

lambda $\lambda(t)$ is obtained using an optimization cost function based on the combustion engine operating point. The results of the ECMS model using the variable lambda will be analysed and discussed.

\subsubsection{Optimization method}

The optimization routine is based on minimizing the difference in combustion engine operating points $\left(\omega_{e}, T_{e}\right)$ between the simulation model and the measured data. The speed and torque of the combustion engine together with the requested vehicle speed and drive power determine all the powers, speeds and torques in the transmission. Consequently, also the battery state-of-energy is determined by the engine operating point. Minimization of the difference in engine torque and speed between the ECMS model and the measured vehicle data will result in an accurate fit for all the powers, torques and speeds in the transmission. The outline of the minimization method is depicted in Figure 10.

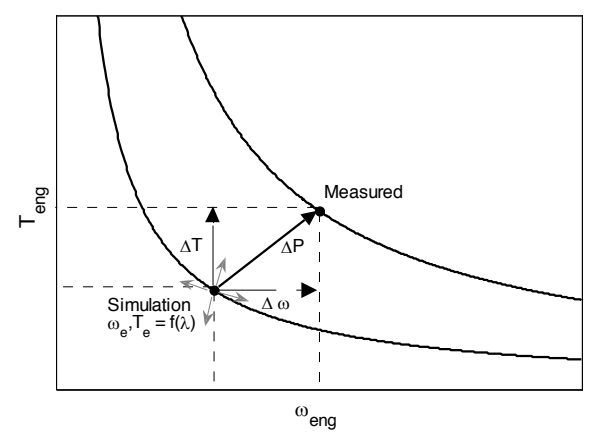

Figure 10: Illustration of outline of the used minimization method.

The engine operating point of the ECMS simulation model can be controlled by variation of the equivalent fuel cost factor $\lambda$. Therefore, $\lambda$ is used for minimizing the difference in engine torque $\Delta T$ and engine speed $\Delta \omega$. This is done by 
minimizing the difference in engine power $\Delta P$, which can be written as (see, also Figure 10):

$\Delta P=\sqrt{\Delta \omega^{2}+\Delta T^{2}}$.

The simulation model is analysed using a vector of values for the equivalent fuel cost factor. At each time instant $t$ on the drive cycle, $\Delta P$ is determined as a function of $\lambda$ using a cost function denoted with $J$. The cost function $J$ as a function of time and $\lambda$ is defined as:

$$
J(t, \lambda)=\sqrt{\left(T_{e}^{S}(t, \lambda)-T_{e}^{M}(t)\right)^{2}+\left(\omega_{e}^{S}(t, \lambda)-\omega_{e}^{M}(t)\right)^{2}}
$$

where $S$ denotes Simulation model and $M$ the measured data. The optimal equivalent fuel cost factor at each time point in the drive cycle $\lambda(t)$ is determined by minimizing the difference in engine power $\Delta P$ and thus by minimizing cost function $J$ for each time step in the drive cycle:

$$
\lambda(t)=\arg \min _{\lambda \in L} J(t, \lambda)
$$

where $L$ is the vector of values of $\lambda$ that was analysed. Using $\lambda(t)$ and the corresponding engine speed $\omega_{e}^{S}(t)$ and engine torque $T_{e}^{S}(t)$, all the other speeds, torques and powers in the transmission and the battery energy are determined.

\subsubsection{Simulation results using a variable $\lambda$}

The optimization routine based on minimizing the difference in engine torque and speed between the ECMS simulation model and the measured vehicle by variation of the equivalent fuel cost factor $\lambda$ is performed for the three different drive cycles (see, Figure 5). The variable equivalent fuel cost factor $\lambda(t)$ obtained with the optimization routine is illustrated for the different drive cycles in Figure 11. Figure 11 shows that the $\lambda(t)$ that follows from the optimization routine varies strongly from the optimal constant equivalent fuel cost factor that was obtained in Section 4.1. The optimal variable factor $\lambda(t)$ corresponds to an optimized combustion engine operating point for the simulation model. The engine torque and speed that follow from the optimization routine are shown in Figure 12. The engine torque and speed from the measured vehicle data and the simulation model using a constant equivalent fuel cost factor are also indicated in the same figure. The results in Figure 12 show that the use of a variable equivalent fuel cost factor improves the accuracy of the ECMS model. The fit of the engine torque and speed of the simulation model with the measured values is significantly improved with respect to the use of a constant equivalent fuel cost factor.
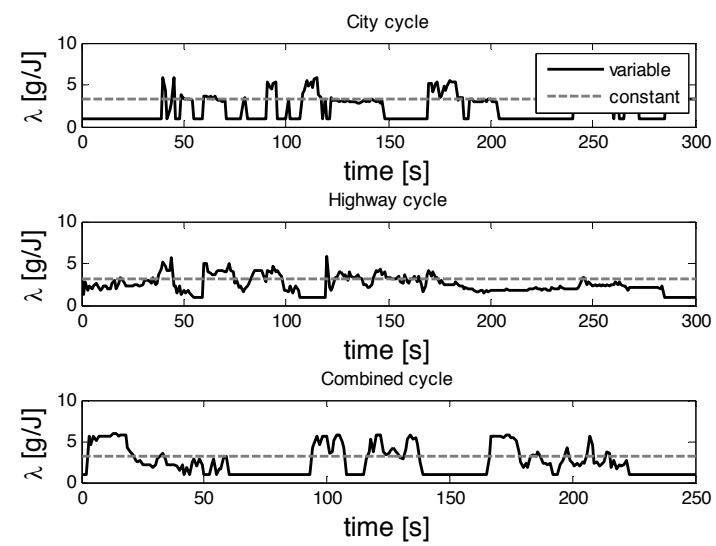

Figure 11: Variable and constant equivalent fuel cost factor for different drive cycles.
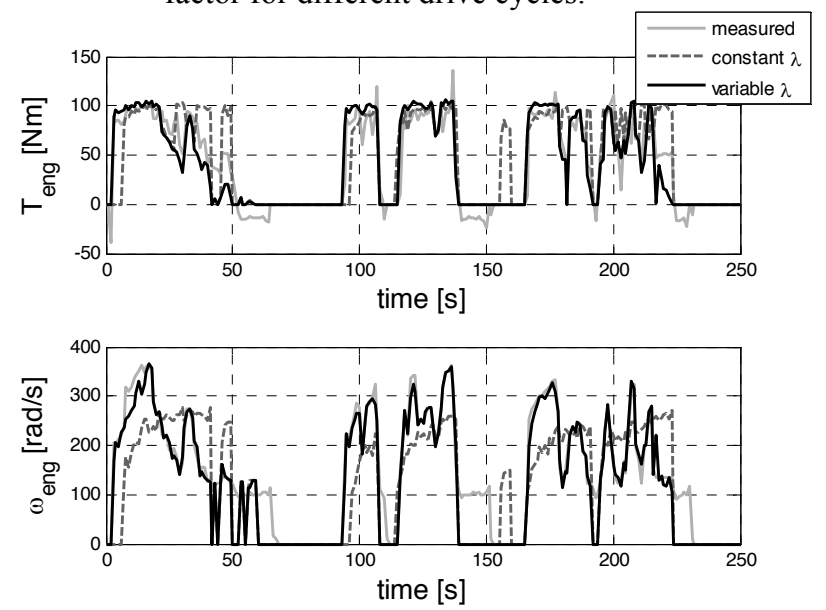

Figure 12: Engine torque and speed for constant and variable equivalent fuel cost factor, and for the measured vehicle.

The improved fit of the simulation model engine torque and speed also affects the accuracy of the other transmission signals. This is shown in Figure 13 , where the engine power and battery energy are plotted for the simulation model using variable, constant equivalent fuel cost factor, and for the measured vehicle data. The accuracy of the simulation using a variable $\lambda$ is significantly improved with respect to the constant $\lambda$ simulation model. 

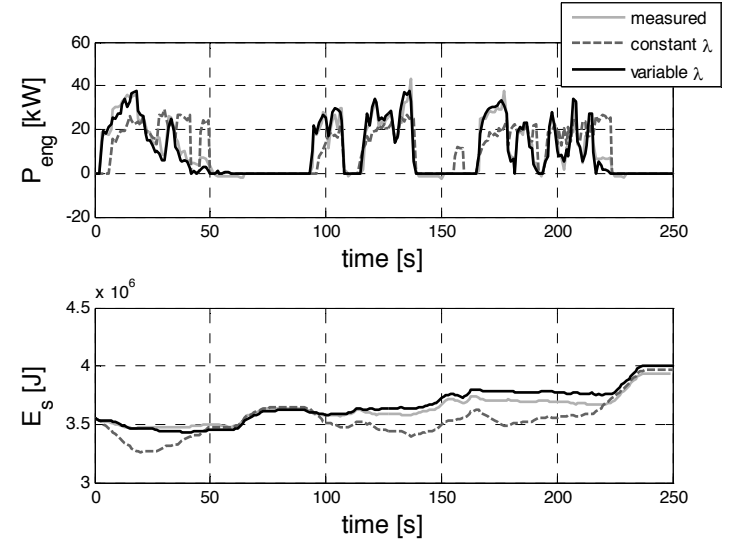

Figure 13: Engine power and battery energy for the simulation model using a constant and variable equivalent fuel cost factor, and for the measured vehicle.

In Table 4 the fuel consumptions results are listed for the simulation model using a variable and constant $\lambda$, and for the measured vehicle. In contrary to usage of a constant $\lambda$, it can be observed that the fuel consumption for the city cycle using a variable equivalent fuel consumption factor differs significantly less from the measured fuel consumption $(+3.4 \%)$. For the combined cycle, the results are the same for both constant and variable $\lambda$ (-4.4\%). On the highway cycle, the variable $\lambda$ results in significantly lower fuel consumption compared to the measured vehicle $(-14.6 \%)$. This is caused by limitations in the optimization routine and by differences in energy management strategy.

Table 4: Fuel consumption for the simulation model using constant and variable $\lambda$ and for the measured vehicle

\begin{tabular}{lccc}
\hline & \multicolumn{3}{c}{ Fuel consumption $[1 / 100 \mathrm{~km}]$} \\
\cline { 2 - 4 } & City & Highway & Combined \\
\hline $\begin{array}{l}\text { Simulation model, } \\
\text { constant } \lambda\end{array}$ & 4.83 & 5.57 & 5.42 \\
$\begin{array}{l}\text { Simulation model, } \\
\text { variable } \lambda\end{array}$ & 7.51 & 4.77 & 5.42 \\
$\begin{array}{l}\text { Measured THS II } \\
\text { vehicle }\end{array}$ & 7.26 & 5.59 & 5.67 \\
\hline
\end{tabular}

\subsubsection{Discussion}

However, differences in engine operating points between the variable $\lambda$ simulation model and the measured vehicle data still occur. These differences originate from the principle of the ECMS and the use of the equivalent fuel cost factor as a control variable. The engine operating point of the ECMS simulation model is controlled by variation of $\lambda$. For example, in Figure 14, the engine operating points at a time instant $(t=20 \mathrm{~s})$ on the combined cycle for various values of $\lambda$ are shown. The optimal engine operating point is also indicated (circle), as well as the measured engine operating point (square).

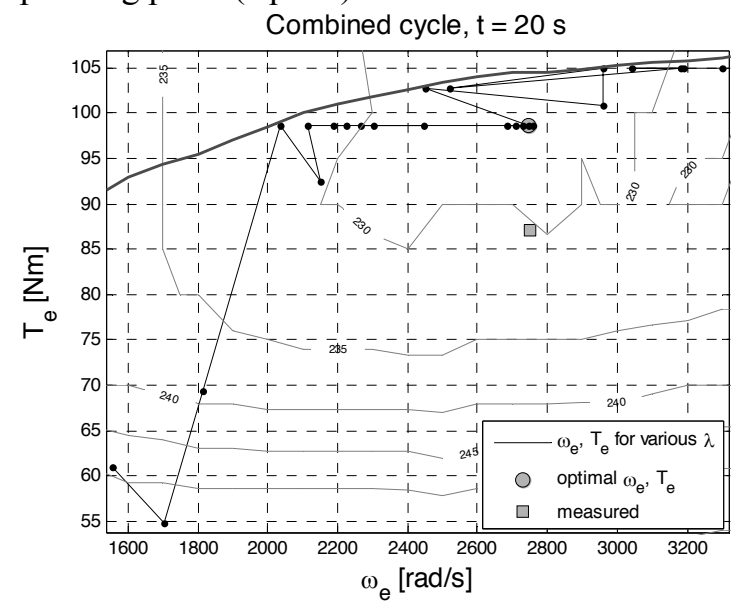

Figure 14: Influence of the equivalent fuel cost factor on engine operating point.

It can be observed that the use of a variable equivalent fuel cost factor does not guarantee an accurate fit with the measured data. The variable $\lambda$ does not provide enough control freedom to position the engine operating point in the desired measured operating point. The variable $\lambda$ is used for controlling two system states, the engine torque and engine speed. A compromise is made between these two states for finding the optimal lambda. Furthermore, ECMS always provides an optimal solution for the control of the engine operating point, independently of the value of $\lambda$. Therefore, it is not possible for the ECMS simulation model to operate in a non-optimal point, whereas the THS II could prefer this non-optimal engine point for various other yet unknown reasons (e.g., low battery state-of-energy, temperature, and life time issues) which are not incorporated in the simulation model. 


\section{Conclusions}

The Energy Management Strategy (EMS) of the THS II is analyzed using measurement data collected with a Toyota Prius under different driving conditions on the road and compared with an optimized EMS based on simulations. The ECMS method uses an equivalent fuel consumption factor for computing the optimal engine operation points while satisfying the energy balance and other defined drive train constraints. Therefore, the optimal variable equivalent fuel cost factor $\lambda$ is determined using an optimization routine that is based on minimizing the difference in engine torque and engine speed between the simulation model and the measured vehicle. The analysis of the use of a variable equivalent fuel cost factor $\lambda$ leads to the following conclusions: (i) the use of a variable equivalent fuel cost factor $\lambda$ in the ECMS simulation model leads to results significantly closer to the measured vehicle data, compared to the use of a constant equivalent fuel cost factor. (ii) However, the use of a variable equivalent fuel cost factor $\lambda$ in the ECMS simulation model does not significantly improve the fuel consumption, compared to the use of a constant equivalent fuel cost factor.

For each analysed drive cycle, the battery stateof-energy behaves significantly more constant than for the ECMS simulation model. It is found that the EMS of the Toyota Prius tends to focus more on minimization of the battery power in power-split mode. The ECMS focuses on minimizing the fuel consumption.

The use of ECMS with a constant $\lambda$ in a Toyota Prius leads to an improvement in fuel consumption of $36 \%$ in city traffic, $2.5 \%$ in highway traffic and $7 \%$ in combined traffic compared to the measured vehicle data.

\section{References}

[1] J. Liu, H. Peng and Z. Filipi, "Modeling and Analysis of the Toyota Hybrid System", in International Conference on Advanced Intelligent Mechatronics 2005

[2] R. H. Staunton, C. W. Ayers, L. D. Marlino, J. N. Chiasson, T. A. Burress, "Evaluation of 2004 Toyota Prius Hybrid Electric Drive System", May 2006

[3] J.T.B.A. Kessels, "Energy Management for Automotive Power Nets", PhD Thesis, Eindhoven University of Technology, February 2007

[4] J.M. Miller, "Hybrid Electric Vehicle Propulsion System Architectures of the e-
CVT Type", IEEE Transactions on power Electronics, Vol. 21(3), May, 2006

[5] J.T.B.A. Kessels, M.W.T. Koot, R.M.L. Ellenbroek, M.F.M. Pesgens, F.E. Veldpaus, P.P.J. van den Bosch, M. Eifert, D.B. Kok, "Vehicle Modeling for Energy Management Strategies", in Proc. of the $7^{\text {th }}$ International Symposium on Advanced Vehicle Control, Arnhem, 2004

[6] J. S. Hsu, C. W. Ayers, C. L. Coomer, "Report on Toyota/Prius Motor Design and Manufacturing Assessment”, ORNL/TM2004/137

[7] K. Muta, M. Yamazaki and J. Tokieda, "Development of New-Generation Hybrid System THS II - Drastic Improvement of Power Performance and Fuel Economy", SAE paper, 2004

\section{Authors}

T. (Theo) Hofman received his M.Sc.(with honors) and Ph.D.-degree in Mechanical Engineering from

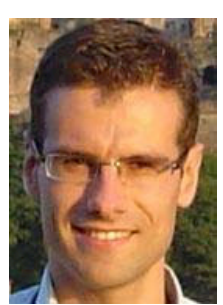
Eindhoven University of Technology, Eindhoven. Since September 2007, he is a post-doctoral fellow with the Control Systems Technology group. His research interests are modeling, design, and control of hybrid and electric technologies for propulsion systems.

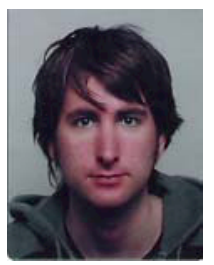

T. (Thijs) Purnot received his B.Sc.degree in Mechanical Engineering from Eindhoven University of Technology, Eindhoven, The Netherlands, in 2006. 